\title{
An Intelligent Path Evaluation Algorithm for Congestion Control in Wireless Sensor Networks
}

\author{
Makul Mahajan ${ }^{1}$, Dr. Sukhkirandeep Kaur ${ }^{2}$ \\ ${ }^{1}$ Research Scholar, Lovely Professional University, Punjab \\ ${ }^{2}$ Assistant Professor, Lovely Professional University, Punjab
}

Article History: Received: 10 November 2020; Revised 12 January 2021 Accepted: 27 January 2021; Published online: 5 April 2021

DOI: https://doi.org/10.17762/turcomat.v12i6.6953

\begin{abstract}
In Wireless Sensor Networks (WSNs), congestion occurs when the traffic load on the node reaches obvious network channel power and is considered as a prominent problem. So it is highly essential to identify, prevent and fix congestion when taking into account the network's restricted re- sources. If congestion in the network is not managed correctly, it can lead to significant loss of QoS parameters, such as decreasing network throughput, increasing energy usage, and decreasing the distribution ratio of packets at the destination or sink node. In this article, we have formulated an algorithm to manage congestion using a modified ant colony algorithm that considers the residual energy, buffer occupancy and bandwidth of the neighbour node for selecting the best node to route the traffic that needs strict QoS requirements. For routing the traffic, there may be a variety of neighbour nodes exists in the network , and if congestion occurs at any selected neighbour node the algorithm will rework to check the quality of rest of the neighbor nodes by calculating a path preference probability metric. The neighbour node with maximum path preference probability will be selected for routing the data packets belonging to realtime traffic that have stringent QoS. The proposed algorithm was simulated and tested using NS 2.33 and it was found that as opposed to the standard AntSense algorithm, the proposed algorithm is capable of achieving lower average energy consumption and packet loss ratio respectively with higher throughput.
\end{abstract}

Keywords: Congestion, Average Energy consumption, packet loss ratio, throughput, ant colony optimization

\section{Introduction}

\subsection{Wireless Sensor Network}

WSNs are simple computers with a variety of devices mounted on them that are used to configure environmental as well as physical data such as pressure, sound, orientation, vibration, and temperature, amongst many other factors. The input obtained from the sensors is redirected from one sensor to another via a multi-hop routing protocol to access the sink node. The base station is in possession of the results aggregation and analysis. Sensor nodes in WSNs have limited resources in terms of power consumption, resources, storage, bandwidth, processing capabilities, and so on. Initially, the researchers managed to come up with a routing scheme that would enable them to make efficient use of WSN resources. Nodes are mostly self-organized and connect with each other via wireless networks, which are implemented throughout the WSN on an ad hoc basis. The main obstacles amongst the other possible problems is that the routing mechanism of the WSN can be compromised, culminating in inevitable losses due to network congestion. In order to provide the congestion-free efficient routing, a number of techniques based on Ant colony optimization have been proposed (Akyildiz et al., 2002; Akyildiz et al.,2002). However, most of the techniques are either using residual energy or the gap between the node, and its destination is used to measure the likelihood of packet transmission using a neighbour node. So, in order to design an efficient congestion control algorithm, we have taken residual energy of the neighbour node, buffer occupancy and bandwidth allocated for calculating the probability of the next neighbor node for selection. The remaining section of this paper is as follows: Section 2 is a comprehensive review of related work, Section 3 is the basic Ant colony optimization work, Section 4 is the proposed Ant colony optimization algorithm, and Section 5 is the simulations and analysis of the results. Finally, the paper will be finalized in Section 6.2.

\subsection{Congestion in Wireless Sensor Networks}

Congestion is the most prominent issue in WSNs that adversely affect the network QoS parameters, must be handled properly. When the multiple sensor nodes start sending the data simultaneously the data generated at intermediate nodes increases. As a result, the number of data packets and generated data from the source nodes exceeds the underlying capacity of the network queue and at the same time makes the networks underperforming 
due to congestion. In WSNs, we can have a variety of causes for congested networks, such as overloading of the queue, collision of packet with each other, parallel transfer of data packets sharing the bandwidth etc.There are 2 levels at which congestion can occur in the networks: node-level congestion and link-level congestion. Nodelevel congestion is very common in both conventional and wireless sensor networks. The key cause for nodelevel congestion is buffer overload at the sink or intermediate node in the network. Congestion at node-level contributes to packet loss and increased queuing delays. On the other hand, as more than one sensor node tries to transfer data within the same range, this leads to congestion at the link-level. In inclusion to growing network losses and wastage of network resources like energy, this type of congestion has an unfavorable impact on the throughput and connection capacity of the network (Wang et al.,2007; Patel et al.,2013). A sophisticated and accurate congestion management system is needed to make the network efficient. There are sections of congestion management: the detection of congestion, the confirmation of congestion and the control of congestion outlined below.

\subsubsection{Congestion Detection}

In the Wireless Sensor Networks, the role of sensing congestion and locating a scene of congestion is to predict congestion. Various metrics are used to track interference in wireless sensor networks: packet losses, Buffer occupancy and channel load.

- Packet loses: Packet losses can be estimated using ACKs on the sender side, the sequence number can be used on the receiver side; consistent packet loss can be used as an illustration of congestion.

- Buffer occupancy: This congestion detection parameter is also recognized as the length of the queue. Each WSN system retains a buffer for storing incoming packets if they cannot be transmitted immediately. Algorithms that use this parameter to detect congestion usually use a constant buffer threshold. As the length of the queue or the occupancy of the buffer raises the constant threshold value, congestion is identified, and some warning is raised.

- Load on a network channel load or busyness: can be another metric to detect congestion. Packet load is measured in the wireless medium of communication, and congestion is identified when the packet transmission time reaches the predefined threshold time. When the channel is occupied, the packet collision will increase. In the case of a spike in packet collision, several packets are eliminated from the buffer and thus the occupancy of the buffer decreases (Patel et al.,2013; Sergiou et al.,2014).

\subsubsection{Congestion Notification}

It is the obligation of the protocol operating in the network to transmit congestion- based information to expecting nodes so that effective ventures can be taken. These details may be a bit of congestion or a control message. Explicit congestion refers to data that is sent as a data traffic jam warning bit in the incoming packets, while implicit congestion refers to data that is sent as a separate surveillance code.

- Explicitly notification of congestion: In this strategy, the source node is intimated as to congestion by the use of control packets in the event of congestion detection. Sending the congestion condition in the form of control packets to the already congested network puts an extra burden on the network. This approach is therefore not commonly used and used by some of the congestion management protocols. This is the major drawback of being specifically told of congestion (Sergiou et al.,2014; Tao et al.,2010).

- Implicitly notification of congestion: Implicit congestion warning strategy does not use control packets to monitor congestion to the respective source nodes and load on the already congested nodes and the network. The packet payload header is piggybacked with congestion information and distributed to other nodes on the network (Sergiou et al.,2014; Tao et al.,2010).

\section{Related Work}

In real-world scenarios, there is a number of optimization problems exists which are difficult to solve and categorized as NP-hard category problems. The two basic categories of techniques that can be used to solve such kind of problems are: Providing the actual algorithm and the approximate results algorithms which optimally generate the approximate results through a sequence of iterations. The main difference between the two techniques is that the former one will provide us with the optimal solutions with more computation time, but the later one will use less computation time for generating the optimal results for NP-hard problems.

The routing problem based on Ant Colony Optimization (ACO) is a category of NP-hard approach that is implemented in WSN in particular. ACO is an algorithm based on swarm intelligence, motivated by the discovery behaviour of ant colonies present in the natural environment, and is a powerful technique to fathom combinational optimization algorithms. A multi-pronged decomposition-based ant colony algorithm (Cheng et 
al.,2012) was put forwarded, where the colony of the ant was split into several subspecies , and for each group, one pheromone pattern was kept. However the importance of separate objectives for all targets to stake the equivalent pheromone data could not be expressed in this approach, which meant that the objective functions were similarly relevant. In order to solve the problem of multiple targets of differing meaning (Hackel et al., 2008), a Pareto optimization approach hinged on dynamic programming with multilevel of pheromone and heuristic intelligence was proposed. With the help of two pheromone matrices and a probing matrix (Prasad et al. 2013), a routing scheme with Multi-objective and multi cast criteria based on the ant colony algorithm was used to route packets with contended QoS. However, none of the algorithms mentioned above has not considered the congestion while routing the data from one node to another node.

A mobile opportunistic network was introduced by (Liu et al., 2014), which guarantees an autopoiesis network that does not requires the absolute information of the source and destination path. An algorithm was suggested by (Ming-Hua et al., 2012), which applies the concepts of fuzzy logic on ant Colony optimization algorithms called algorithm for Fuzzing Ant Colony Optimization (FACOA). The algorithm will calculate parameters like residual and energy pheromone through three phases: fuzzing, conjecture, and de-fuzzing. Finally, due to the consequence of fuzzy selection, the ants selected the next hop, and the delay-tolerant network was introduced by (Xiao et al., 2016) and the data transmission scheme was proposed. (Begam et al. 2010) concentrated on optimization of the route in the genetic techniques and the conventional ant colony approach are based on WSN. Nonetheless, the topic of use of electricity is underestimated. (Sun and Tian, 2010) illustrated the slow rate of convergence and the long starting time of the standard algorithm based on an ant colony On the genetic algorithm and suggested a way of splitting the ants in the reverse direction into two classes In the course of route finding. As a consequence, the rate of convergence increased.

(Sun and Tian, 2010; Orojlo et al., 2012;Tong et al., 2015), From an energy optimization perspective, improved the conventional ant colony algorithm, but the algorithm's complexity is high and a lot of needless calculations are carried out.

In order to consider the factor of congestion in the routing algorithm we have designed a novel algorithm for routing the information from optimal path. The main contributions of the article are as follows.

- To evaluate the various paths available for routing the information from neighbour node to the next node. Path evaluation will be done by calculating the path preference probability of the neighbor nodes using Ant colony opti- mization probability function and selecting the neighbor node with maximum path preference probability.

- To consider the residual energy of neighbour node, bandwidth allocated and buffer occupancy for calculate the path preference probability.

- To simulate the proposed algorithm by using Ns-2.33 simulator and analyzing the results on network parameters throughput, average energy consumption and packet delivery fraction. Finally comparing the results of the proposed algorithm with the traditional Antsense algorithm.

\section{Traditional Ant Colony Optimization}

Met heuristic is a collection of dedicated rules that can be utilized to establish heuristic procedures that apply to a large variety of different optimization problems. (Sharma et al., 2016) has formalized ACO as a met heuristic for mixture optimization problems. ACO takes inventiveness from the provender practices of ant species who inject pheromones on the ground to signify any suitable routes that other members of the colony should follow. To solve problems of optimization, ACO uses a similar mechanism. An individual sensor is insect type species with minimal memory requirement and involved in executing simple ventures. The colony communicates a diverse collective activity that provides an intelligent process to the problems such as moving heavy items, building bridges and discovering the quickest pathways from the nest to the source of food, since a unique insect has no broad understanding of the function it plays and its activities are based on local settlements and are highly irregular. This intelligent behaviour of course occurs as a result of self-sustaining and incidental communication between ants, which can be referred to as (Sharma et al., 2014; Camilo et al.,2006) swarm intelligence.

In the basic part of ACO algorithm the process iterates through three steps after initialization: ConstructAntSolutions, ApplyLocalSearch algorithm, and UpdatePheromones process. A series of surrogates are established by ants that are optimally improved by a local search algorithm and the update pheromone process. In view of this formulation, by moving simultaneously and asynchronously to a sufficiently defined graphical representation that represents any given problem domain, ACO ants produce solutions to the problem. Ants select the next neighbour hop node $\mathrm{j}$ from a node $\mathrm{i}$ according to the probability formula defined given below (Sharma et al.,2016) 


$$
P_{i, j}=\frac{\left[T_{i, j}\right]^{\alpha} *\left[D_{s, d}\right]^{\beta}}{\sum_{n \in N_{i}}\left[T_{i, j}\right]^{\alpha} *\left[D_{s, d}\right]^{\beta}}
$$

where $\mathrm{Pi}, \mathrm{j}$ is the probability of ant selecting the next node $\mathrm{j}$ from node $\mathrm{i}$. $\mathrm{Ti}, \mathrm{j}$ is the concentration of pheromone in the path $\mathrm{i}$ to $\mathrm{j}$. Ni indicated the number of next neighbour nodes that an ant can select. $\alpha$ indicates the weight of the concentration of pheromone. $\mathrm{Di}, \mathrm{j}$ is the distance of next neighbour node from $\mathrm{i}$ node and $\beta$ indicates the weight of the distance.

Wireless sensor networks routing is a difficult problem since the features of the network, such as traffic loading and network topology, can differ stochastically and in a time of varying nature. The distributed design of network routing is well associated with the multi-agent nature of ACO algorithms. Traffic- adaptive and multipath routing is given by the collection of unique properties that characterises ACO objects for routing problems, based on both passive and active data tracking and collection, using hypothetical units, not allowing local processes to have a global effect, setting up routes in a less greedy way than in strictly shortest path techniques preferring balancing of network load (Sharma et al.,2016).

\section{Proposed Work}

In the suggested algorithm, Path evaluation will be done using the intelligent behaviour of ANT colony optimization algorithm and congestion will be controlled, if occurs, by routing the different type of traffic from different paths based upon the goodness of paths available. Congestion in the network will be detected at the node level by checking the buffer occupancy of the node. The proposed algorithm is based on devising the algorithm for evaluating the best path for real-time traffic like audio/video using an updated Ant colony optimization algorithm. For this purpose algorithm will identify if there is congestion in the network by checking the buffer occupancy of the neighbor node for transferring the packets. If buffer occupancy falls under the situation that can lead to congestion, we need to identify the packets belonging to different traffic types like audio, video or scalar traffic. The packets belonging to real-time traffic will be routed through the best node among the neighbors. In sensor networks, there can be variety of sensor nodes generating the data packets which may belong to scalar as well as real-time traffic. The main objective of the proposed algorithm is to route the real time packets through the nodes having maximum path preference probability.

In the event of congestion, the proposed algorithm will calculate the path preference probability of all the neighbour nodes from where data can be routed. The path preference probability will be calculated by considering the residual energy, bandwidth and buffer occupancy of the neighbour nodes and node with the maximum path preference probability will be selected for routing the information. In order to calculate the path preference probability, we will be using the mathematical model illustrated below. The path preference probability from node $\mathrm{i}$ to neighbour node $\mathrm{j}$ can be calculated using the following mathematical function.

$$
P p F_{i, j}=\frac{\left(E_{i, j}\right)^{\alpha} *\left(B_{i, j}\right)^{\beta} *\left(B O_{i, j}\right)^{\gamma} *\left(T_{i, j}\right)^{\theta}}{\sum_{n \in N_{i}}\left(E_{i, j}\right)^{\alpha} *\left(B_{i, j}\right)^{\beta} *\left(B O_{i, j}\right)^{\gamma} *\left(T_{i, j}\right)^{\theta}}
$$

where $\mathrm{PpFi}, \mathrm{j}$ is the path preference probability of ant selecting the next node $\mathrm{j}$ from node $\mathrm{i}$. Ni indicated the number of next neighbor nodes that an ant can select. Ei,j is the energy of next neighbor node from $i$ node and $\alpha$ indicates the weight of energy. Bi,j is the bandwidth of next neighbor node from i node to node $\mathrm{j}$ and $\beta$ indicates the weight of bandwidth. BOi,j is the buffer occupancy of the node $\mathrm{j}$ and $\gamma$ indicates the weight of buffer occupancy. $\mathrm{Ti}, \mathrm{j}$ is the accumulation of pheromone in the path $\mathrm{i}$ to $\mathrm{j}$ and $\theta$ indicates its weight.

The Algorithm flowchart of the proposed algorithm is shown in figure 1 


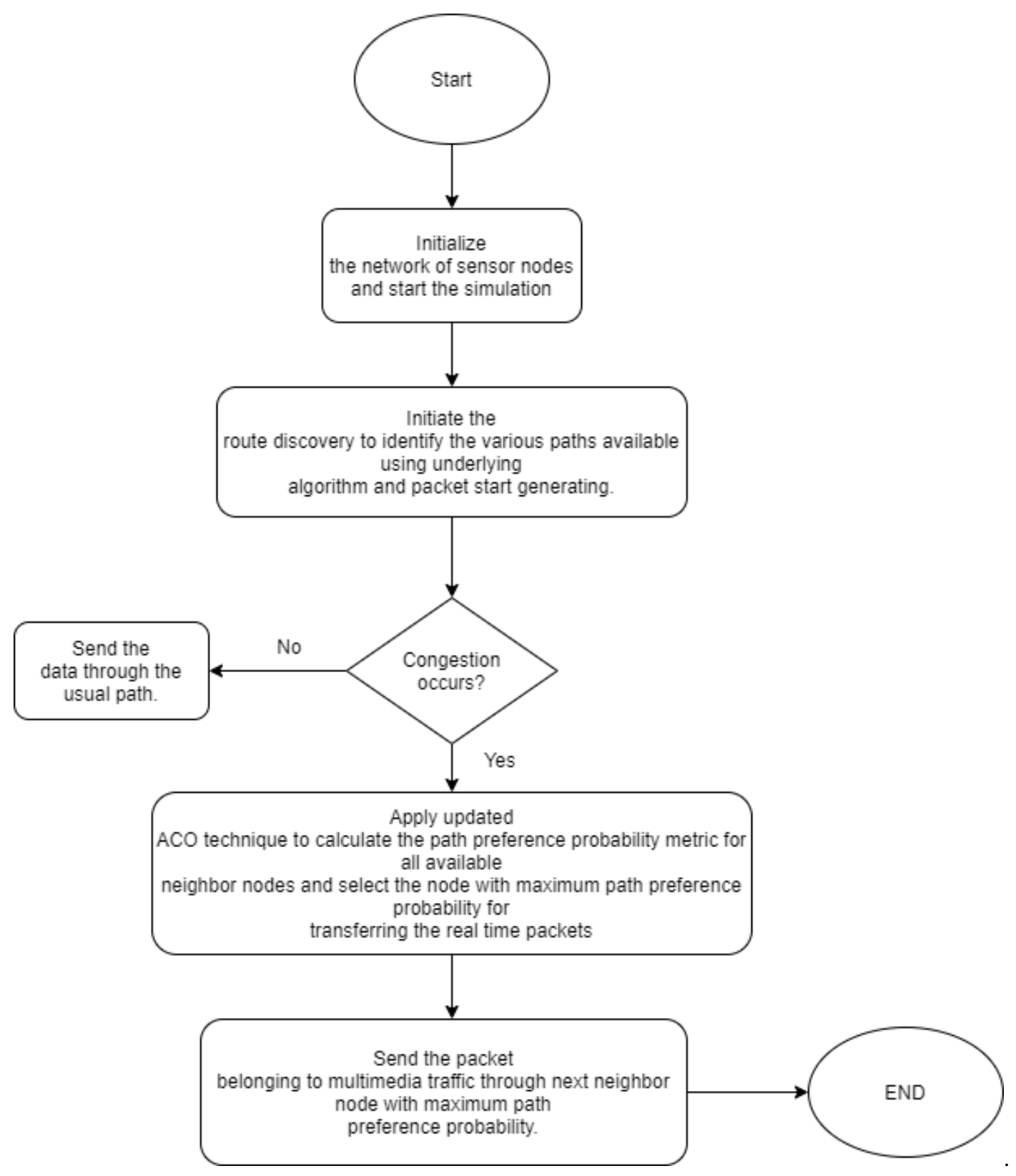

Figure 1: Algorithm flowchart of proposed algorithm

\section{Simulation and Result Analysis}

Proposed algorithm using updated ant colony optimization algorithm and traditional algorithm Antsense under the situation of congestion have been simulated by using a well-known simulator NS-2.33 under the varying number of sensor nodes. A number of extensive simulations have been performed to check the efficacy of the developed algorithm. Nodes are put randomly in a $2000 \mathrm{~m}$ x $2000 \mathrm{~m}$ zone in all scenarios. Each mobile node's maximum transmitting range is $100 \mathrm{~m}$. Static nodes are called the sensor nodes that have been simulated on the network. The channel bandwidth is $2 \mathrm{Mbps}$ and the IEEE 802.11 DCF is used for the MAC layer. Each simulation runs for 100 seconds at a time. A variety of CBR sources with multiple sources for the development of multiple traffic types and with a single destination have generated data traffic. Various parameters taken for the network simulation is listed Table 1. 
Table 1: The simulation parameters.

\begin{tabular}{ll}
\hline Simulation Secenario and paramters & Corresponding value \\
\hline Wireless propagation model & TwoWay around \\
Node's Transmission range & $100 \mathrm{~m}$ \\
Queue Type & PriQueue \\
Node's initial energy & $90 \mathrm{~J}$ \\
Simulation Time & $100 \mathrm{~s}$ \\
Packet's Size & 1024 Bytes \\
Antenna Pattern Used & OmniAntenna \\
\hline
\end{tabular}

\subsection{Average Energy Consumption Analysis}

The figure 2 represents the average energy consumption at the end of simulation and it has been observed that the proposed algorithm shows less energy consumption as compared to traditional Antsense algorithm. This is due to the fact that the proposed algorithm uses bandwidth between the two nodes as well as buffer occupancy of the neighbor node to be selected for packet transmission in addition to residual energy of the node. As shown in the figure extensive simulations have been performed by varying the number of nodes in the network and in all the scenarios the proposed algorithm is showing the positive result. In the event of congestion in the network simulation, the proposed algorithm efficiently able to select the best node among the set of neighbor nodes with maximum path preference probability. Table 2 summarizes the throughput obtained for proposed and traditional Antsense algorithm by varying the number of nodes in the network.

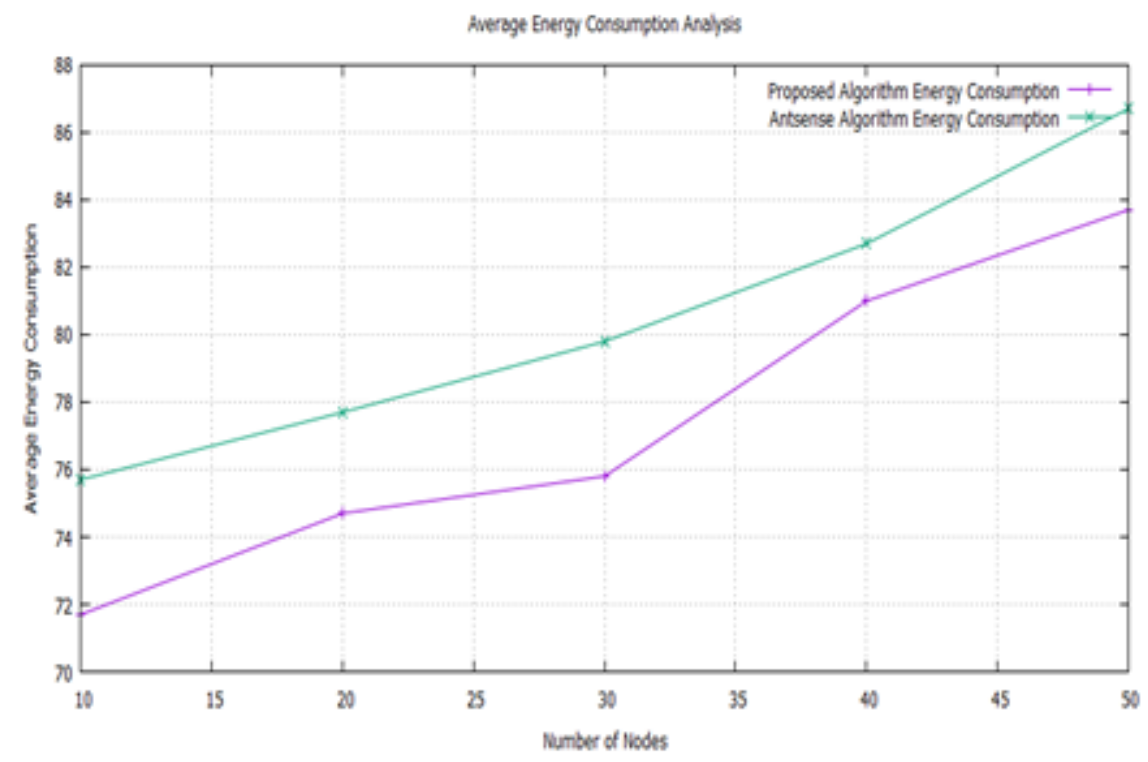

Figure 2: Average energy consumption of proposed algorithm and traditional Antsense algorithm

Table 2 Average Energy consumption of proposed and Antsense algorithm

\begin{tabular}{ccc}
\hline Number of nodes & $\begin{array}{c}\text { Proposed Algorithm Energy } \\
\text { consumption }(\mathbf{J})\end{array}$ & $\begin{array}{c}\text { Traditional Algorithm Energy } \\
\text { consumption }(\mathbf{J})\end{array}$ \\
\hline $\mathbf{1 0}$ & 71.7 & 75.7 \\
$\mathbf{2 0}$ & 74.7 & 77.7 \\
$\mathbf{3 0}$ & 75.8 & 79.8 \\
$\mathbf{4 0}$ & 81 & 82.7 \\
\hline
\end{tabular}




\subsection{Throughput Analysis}

The figure 3 represents the throughput at the end of simulation and it has been observed that the proposed algorithm shows higher throughput as compared to traditional Antsense algorithm. This is due to the fact that the proposed algorithm uses bandwidth between the two nodes as well as buffer occupancy of the neighbor node for selecting the next for packet transmission in addition to residual energy of the node there by able to select the best node for transmission. When the best node is used for transmission among the various available neighbor nodes then the reliability of the network increases thereby increasing the throughput of the network. As shown in the figure 3 extensive simulations have been performed by varying the number of nodes in the network and in all the scenarios the proposed algorithm is showing the positive result. In the event of congestion during the network simulation, the proposed algorithm efficiently able to select the best node among the set of neighbor nodes with maximum path preference probability.

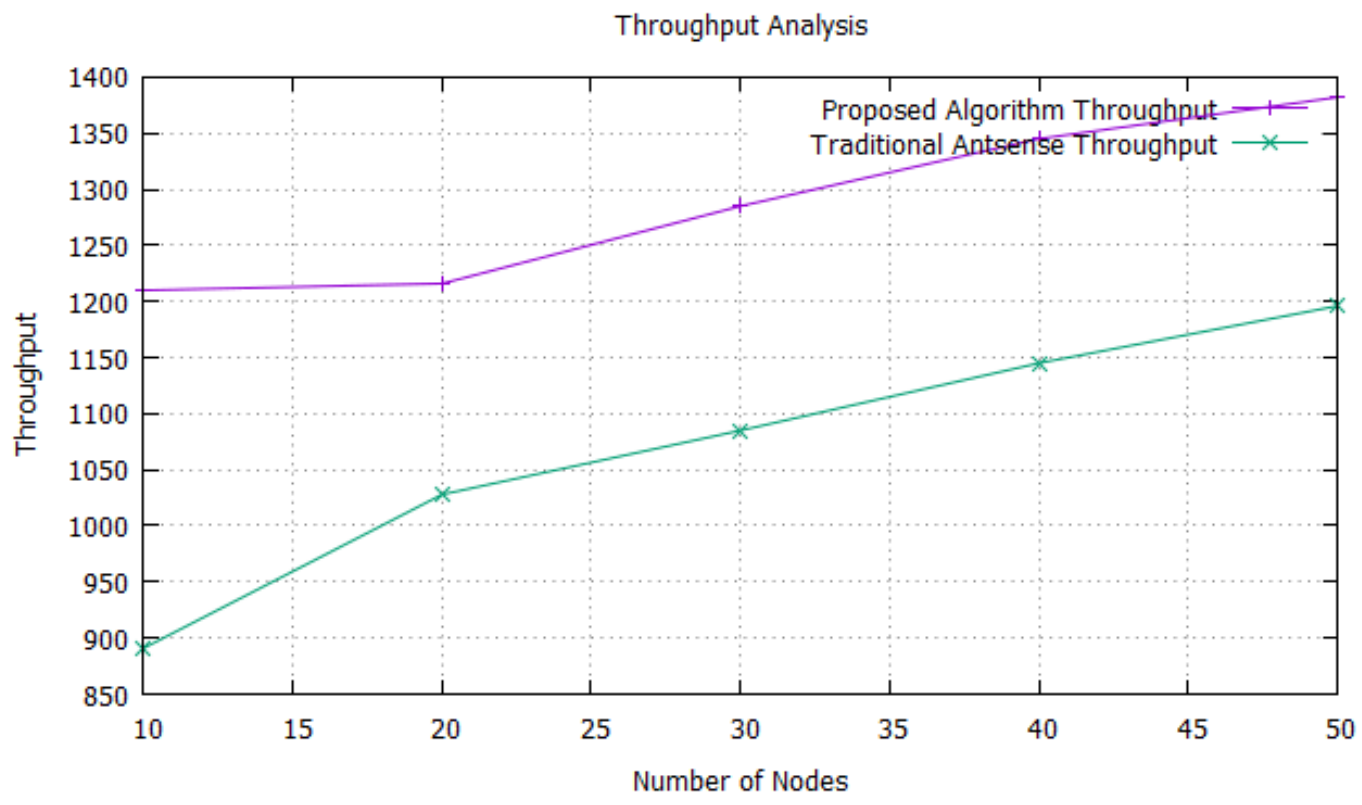

Figure 3: Throughput analysis versus number of nodes for proposed and traditional Antsense algorithm

Table 3 Throughput analysis of proposed and Antsense algorithm

\begin{tabular}{ccc}
\hline Number of nodes & $\begin{array}{c}\text { Proposed Algorithm Throughput } \\
(\text { Kbps })\end{array}$ & $\begin{array}{c}\text { Traditional Algorithm } \\
\text { Throughput (Kbps) }\end{array}$ \\
\hline $\mathbf{1 0}$ & 1210 & 891 \\
$\mathbf{2 0}$ & 1216 & 1028 \\
$\mathbf{3 0}$ & 1285 & 1085 \\
$\mathbf{4 0}$ & 1345 & 1154 \\
$\mathbf{5 0}$ & 1381 & 1196 \\
\hline
\end{tabular}

\subsection{Packet Loss Rate (PLR )}

The Packet loss rate (PLR) is one of the important metric for measuring the performance of traffic related to realtime flows such as video and Voice over IP. The number of lost data packets must be low in order to prove the smooth and seamless data transmission. The number of lost data packets is increasing with the increase in number

of nodes increasing in the simulated network. However, the proposed algorithm has lower packet loss rate increase than that of the AntSense algorithm. This is due to the fact that the proposed algorithm uses the best node among the available neighbor nodes available for data transmission in the event of congestion. When the traffic is 
diverted to the congestion free path from the congested path then packet loss tare decreases as show in the figure 4

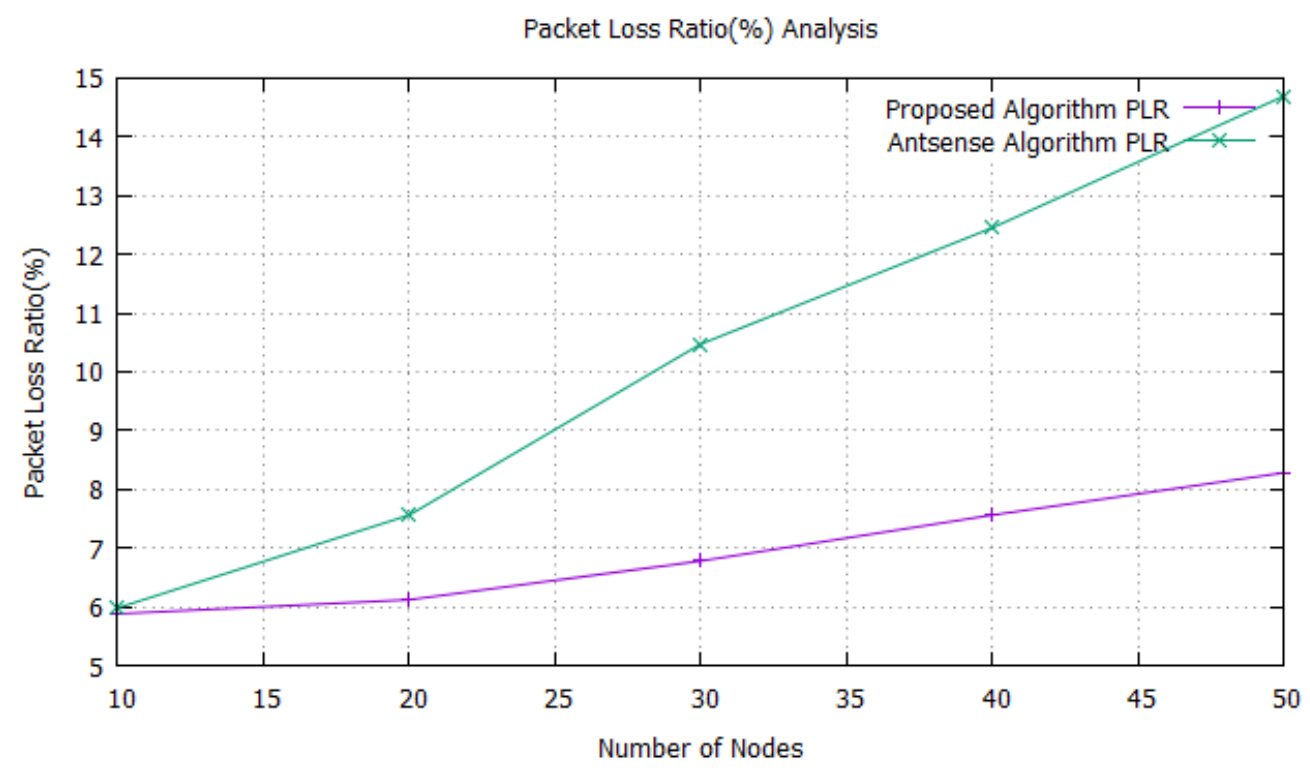

Figure 4: Packet loss rate analysis versus number of nodes for proposed and traditional Antsense algorithm

Table 4: packet loss ratio of proposed and Antsense algorithm

\begin{tabular}{ccc}
\hline Number of nodes & $\begin{array}{c}\text { Proposed Algorithm packet loss } \\
\text { rate (\%) }\end{array}$ & $\begin{array}{c}\text { Traditional Algorithm packet loss } \\
\text { rate (\%) }\end{array}$ \\
\hline $\mathbf{1 0}$ & 5.8 & 6.2 \\
$\mathbf{2 0}$ & 6.1 & 7.5 \\
$\mathbf{3 0}$ & 6.8 & 10.2 \\
$\mathbf{4 0}$ & 7.5 & 12.5 \\
$\mathbf{5 0}$ & 7.8 & 14.5 \\
\hline
\end{tabular}

\section{Conclusion}

In this paper we have proposed a path evaluation algorithm based on modified ant colony optimization technique for controlling the congestion in wireless sensor networks. The proposed algorithm improves the heuristic function for selection the next neighbor node to transmitting the packet. The improved heuristic function considers residual energy, buffer occupancy and bandwidth allocated to the neighbor node and calculates path preference probability of all the neighbor nodes. The algorithm selects the neighbor node with maximum path preference probability. The simulation result shows that the proposed algorithm shows the decrease in the average energy consumption and packet loss ratio and increase in the throughput of the network when compared with AntSense algorithm.

\section{References}

[1]. Akyildiz, I. F., Su, W., Sankarasubramaniam, Y., \& Cayirci, E. (2002). A survey on sensor networks. IEEE Communications magazine, 40(8), 102-114.

[2]. Akyildiz, I. F., Su, W., Sankarasubramaniam, Y., \& Cayirci, E. (2002). Wireless sensor networks: a survey. Computer networks, 38(4), 393-422.

[3]. Begum, S., Tara, N., \& Sultana, S. (2010). Energy-efficient target coverage in wireless sensor networks based on modified ant colony algorithm. International Journal of Ad hoc, Sensor \& Ubiquitous Computing, 1(4), 29-36.

[4]. Cheng, J., Zhang, G., Li, Z., \& Li, Y. (2012). Multi-objective ant colony optimization based on decomposition for bi-objective traveling salesman problems. Soft Computing, 16(4), 597-614. 
[5]. Camilo, T., Carreto, C., Silva, J. S., \& Boavida, F. (2006, September). An energy-efficient antbased routing algorithm for wireless sensor networks. In International workshop on ant colony optimization and swarm intelligence (pp. 49-59). Springer, Berlin, Heidelberg.

[6]. Häckel, S., Fischer, M., Zechel, D., \& Teich, T. (2008, July). A multi-objective ant colony approach for pareto-optimization using dynamic programming. In Proceedings of the 10th annual conference on Genetic and evolutionary computation (pp. 33-40)

[7]. Liu, Y., Yang, Z., Ning, T., \& Wu, H. (2014). Efficient quality-of-service (QoS) support in mobile opportunistic networks. IEEE Transactions on Vehicular Technology, 63(9), 4574-4584.

[8]. Ming-Hua, L., Hua, Z., \& Guang, S. (2012). Energy aware routing algorithm for wireless sensor network based on ant colony principle. JCIT: Journal of Convergence Information Technology, 7(4), 215-221.

[9]. Orojloo H, Moghadam RA and Haghighat AT. WSN path optimization based on fusion of improved ant colony algorithm and genetic Energy and path aware ant colony optimization based routing algorithm for wireless sen- sor networks. In: Krishna PV, Babu MR and Ariwa E (eds) Global trends in computing and communication systems. Berlin; Heidelberg: Springer, 2012, pp.182-191.

[10]. Patel, S. (2013, December). Performance analysis and modeling of congestion control algorithms based on active queue management. In 2013 INTERNATIONAL CONFERENCE ON SIGNAL PROCESSING AND COMMUNICATION (ICSC) (pp. 449-454). IEEE.

[11]. Prasad, S., \& Lobiyal, D. K. (2013, February). Multiobjective multicast routing in wireless ad hoc networks-An Ant Colony approach. In 2013 3rd IEEE International Advance Computing Conference (IACC) (pp. 511-514). IEEE.

[12]. Sergiou, C., Vassiliou, V., \& Paphitis, A. (2014). Congestion control in wireless sensor networks through dynamic alternative path selection. Computer Networks, 75, 226-238.

[13]. Sharma, V., \& Grover, A. (2016). A modified ant colony optimization algorithm (mACO) for energy efficient wireless sensor networks. Optik, 127(4), 2169-2172.

[14]. Sharma, T., Kumar, B., Berry, K., Dhawan, A., Rathore, R. S., \& Gupta, V. (2014, April). Ant based cluster head election algorithm in wireless sensor network to avoid redundancy. In 2014 Fourth International Conference on Communication Systems and Network Technologies (pp. 83-88). IEEE.

[15]. Sun, Y., \& Tian, J. (2010). WSN path optimization based on fusion of improved ant colony algorithm and genetic algorithm. Journal of Computational Information Systems, 6(5), 15911599.

[16]. Tao, L. Q., \& Yu, F. Q. (2010). ECODA: enhanced congestion detection and avoidance for multiple class of traffic in sensor networks. IEEE transactions on consumer electronics, 56(3), 1387-1394.

[17]. Tong, M., Chen, Y., Chen, F., Wu, X., \& Shou, G. (2015). An energy-efficient multipath routing algorithm based on ant colony optimization for wireless sensor networks. International Journal of Distributed Sensor Networks, 11(6), 642189.

[18]. Xiao, F., Xie, X., Jiang, Z., Sun, L., \& Wang, R. (2016). Utility-aware data transmission scheme for delay tolerant networks. Peer-to-Peer Networking and Applications, 9(5), 936-944.

[19]. Wang, C., Li, B., Sohraby, K., Daneshmand, M., \& Hu, Y. (2007). Upstream congestion control in wireless sensor networks through cross-layer optimization. IEEE Journal on selected areas in Communications, 25(4), 786-795. 